\title{
KEY CHALLENGES AND OPPORTUNITIES IN HULL FORM DESIGN OPTIMISATION FOR MARINE AND OFFSHORE APPLICATIONS
}

\author{
Joo Hock ANG ${ }^{1,2}$, Cindy GOH ${ }^{2}$, Yun $\mathrm{LI}^{2}$ \\ 1) Sembcorp Marine Ltd., Singapore 759956 \\ 2) School of Engineering, University of Glasgow, Glasgow, G12 8LT, UK \\ Correspondence email: joohock.ang@sembmarine.com
}

\begin{abstract}
New environmental regulations and volatile fuel prices have resulted in an ever-increasing need for reduction in carbon emission and fuel consumption. Designs of marine and offshore vessels are more demanding with complex operating requirements and oil and gas exploration venturing into deeper waters and hasher environments. Combinations of these factors have led to the need to optimise the design of the hull for the marine and offshore industry. The contribution of this paper is threefold. Firstly, the paper provides a comprehensive review of the state-ofthe-art techniques in hull form design. Specifically, it analyses geometry modelling, shape transformation, optimisation and performance evaluation. Strengths and weaknesses of existing solutions are also discussed. Secondly, key challenges of hull form optimisation specific to the design of marine and offshore vessels are identified and analysed. Thirdly, future trends in performing hull form design optimisation are investigated and possible solutions proposed. A case study on the design optimisation of bulbous bow for passenger ferry vessel to reduce wavemaking resistance is presented using NAPA software. Lastly, main issues and challenges are discussed to stimulate further ideas on future developments in this area, including the use of parallel computing and machine intelligence.
\end{abstract}

Keywords- Simulation-based hull form design optimisation; geometry modeling; shape transformation; performance evaluation; computational fluid dynamic (CFD)

\section{INTRODUCTION}

Increasing environmental regulations and fuel price volatility are two top concerns faced by the marine and offshore industry today. As a consequence, eco-friendly shipping and fuel efficiency are now key design criteria for marine and offshore vessels. Furthermore, the need for bigger vessels and new operating conditions have pushed the frontiers of ship and offshore vessel design beyond conventional design boundaries. For example, as oil and gas exploration ventures into deeper waters and hasher environment, the technical requirements for offshore structures and vessels operating in these environments also increases tremendously. The shape of the hull is tightly coupled to the efficiency of the vessel and has direct impact on its hydrodynamic performance. It is, therefore, a crucial aspect to optimise in order to achieve an overall improvement in vessel performance. Simulation based design (SBD) is widely used in engineering

This research is supported by Economic Development Board (EDB) of Singapore and Sembcorp Marine Ltd under Industria Postgraduate Programme (IPP), grant no: COY-15-IPP/140002 applications and is well-known to improve product performance and design efficiency [1].

Computer-aided design (CAD) tools are commonly used in ship design firms and shipyards for modelling and hydrodynamic evaluation purposes. Simulation-based hull form optimisation, in this case, offers a potential solution to overcome the challenges faced in the design of marine and offshore vessels. This paper will hence focus on the state-of-the-art SBD for hull form optimisation. Section II provides an overview of related works in hull form design optimisation, which includes the key processes and techniques applied. Section III will identify the key challenges for each process and highlight the associated opportunities and key trends. Lastly, a case study of optimisation of passenger vessel bulbous bow for reduced resistance will be presented in section IV, followed by conclusions.

\section{RELATED WORK IN SIMULATION-BASED HULL FORM DESIGN OPTIMISATION}

In the marine and offshore vessel design space, SBD can be used to analyse and improve the hydrodynamic performance of vessels including reduction of resistance and better sea keeping capabilities. Traditional approach to vessel design and testing is a manual and laborious process with a long lead time that could take up to several months to complete. In addition, conventional model test is an expensive process with little tolerance for design error or modifications. Testing can only be done on a single design with minimal variations. A significant advantage of SBD over traditional methods is that it can help shorten the entire design cycle by an appreciable amount. Prior to any physical model testing, SBD can be used to develop 'sufficiently optimum' initial designs virtually which can then be further refined afterwards. The hull form being the largest single component of the entire ship or floating structure exerts the most influence not only on hydrodynamic performance, but also production and subsequently operation of the vessel. It is therefore a crucial aspect to optimise in order to achieve maximum gain on the overall design of the vessel. SBD for performing hull form design and optimisation mainly comprises of 3 key processes namely geometry modelling and manipulation algorithms, optimiser and performance evaluations. These processes can be integrated into a 
common framework for automated hull form optimisation as illustrated in Figure 1.

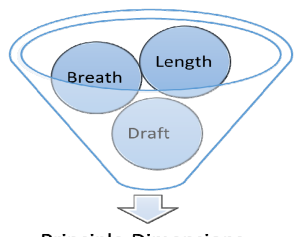

Principle Dimensions
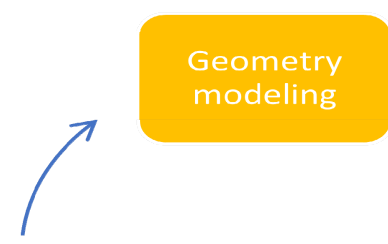

modeling
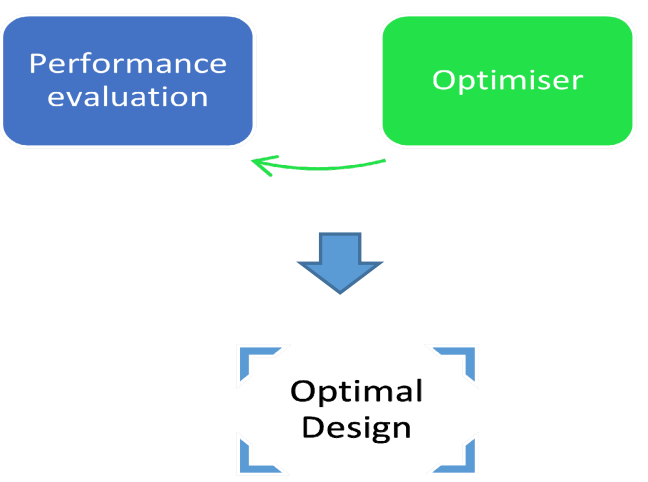

Figure 1. Simulation-based hull form design optimisation framework

\section{A. Geometry Modelling and Shape Transformation}

Geometry modelling of the hull form involves the generation of a geometric shape by first defining in points, followed by representing it using curves before transforming into surfaces. It is an important and integral part of hull form design optimisation and has been studied extensively. Key geometry modelling methods for hull form design optimisation includes Beizer curves, Bsplines and non-uniform rational B-splines (NURBS) [2]. Beizer curves are defined by a set of control points and are in many aspects similar to interpolation. Nonetheless, unlike the latter, it 'stretches' towards, rather than passing through the central control point [3]. B-splines method are developed based on hull form parameters and includes variations such as cubic B splines, fairness optimized B spline or F-splines [4], which helps to overcome the limitation to main algorithm. Due to its ability to accurately represent complex shapes with very small amount of definition data and the ease to manipulate control points, NURBS has become the 'defacto' algorithm for surface representation in hull form geometry design.

After the initial geometry of the hull form is generated, geometry modification or shape transformation methods can then be applied to edit the initial curves and surfaces. In the area of shape transformation, much work has been reported in the literature. Lackenby [5] and 1-Cp are one of the earliest geometry modification methods in hull transformation. It translate the hull section longitudinally using simple calculation and uses simple scaling functions to deform the forward and aft section [6]. Another common method for shape transformation is parametric modelling [7]. By capturing the essence of the intended shapes and the possible variations, it offers better control over the shape to be optimised and can find improved solutions within a shorter timeframe [8]. A popular method for shape transformation of the hull form is Free-form deformation (FFD). Based on the principles of enclosing complex geometric shapes within simpler ones, it provides mapping to change the coordinate position of more complex shape [9]. In hull form design optimisation, it has been applied to transforming the hull design of catamaran [9] and navy vessel [10].

Geometry modelling and shape transformation are complex and delicate processes in hull form optimisation. This is particularly so when an optimal design has multiple objectives to satisfy. Variations to one parameter may improve the performance of one objective at the expense of another, and as a consequence compromise the overall performance of the design. For instance, reducing the input parameters or "control point" while consistently maintaining a smooth surface or 'feasible design' of the hull form are two seemingly conflicting objectives to achieve. It is therefore important to consider the optimisation process as part of the entire SBD framework.

\section{B. Optimisation}

Over the years, SBD has benefited greatly from the developments in optimisation algorithms as well as rapid advancements in computational resources. Suffice to say, there is no one-size-fits-all solution and depending on the objectives and application, the most appropriate optimisation algorithm will be used. In the area of hull form design, the main types of optimisation algorithms are gradient based, deterministic methods, heuristic methods and evolutionary methods. Gradient based methods such as Adjoint equations, Gauss Newton method [11], steepest descent, conjugate gradient [12] and Sequential Quadratic Programming (SQP) [13] have been applied to optimise the hull form of tankers, catamarans and container ship for improved efficiency [14-17].

While these algorithms could be used to determine the characteristics of the search space, such as the extrema, they require information on the search gradient which may not always be available. As a result, the solutions found tend to be sub-optimal. This is particularly so for non-convex problems [1]. They are thus more suitable for local fine-tuning after the proximity of the global maxima or minima has been identified. Deterministic methods are another class of optimisation algorithm that have been applied to improve the design and performance of the ship hull. They include a Hill-climbing technique and Nelder and Mead or downhill simplex method [7]. In [18] and [19], heuristic methods such as fish shoal algorithm (FSA) and Simulated Annealing were applied to optimise the hull of catamaran for reduced resistance and global optimisation model for ship design respectively. Simulated annealing is a stochastic optimisation method 
which is capable of controlling the deterioration of object function so as to escape local minima [19].

In more recent studies, evolutionary algorithms (EA) have become increasingly popular in hull form design optimisation. EA is a class of generic population-based metaheuristic global optimisation techniques [11]. Inspired and modelled closely after biological evolution, it uses mechanisms of selection, recombination and mutation to traverse the search space for fitter solutions. Unlike conventional optimisation algorithms, EA does not make any assumption of the underlying fitness landscape and scales well to higher dimensional problems, making them suitable for NP-complete problems [19]. In hull form design optimisation, commonly used EAs including Genetic Algorithm (GA) [11] and Particle swarm optimization (PSO) [11] have been applied to improve the hydrodynamic performance of ships [20-23].

\section{Performance Evaluation}

Performance evaluation is an integral part of the SBD framework and key to ensure that solutions found are true global optima. It also provides a measure to weed out weaker solutions as the optimisation progresses. In the development of ship hull form, resistance is one of the most important performance parameters that need to be considered. Using linear potential code and Computational Fluid Dynamic (CFD), the evaluation of resistance can be carried out during the early stages of ship design. CFD is a popular tool for evaluating new hull form design. By solving field equations that describes the dynamics of fluid flow, CFD is able to provide accurate simulation of fluid flow [24]. CFD have also been used to assess the performance of vessels in terms of propulsion, seakeeping, maneuvering and propeller designs [25]. Prevalent performance evaluation methods include potential flow and Reynolds Averaged Navier-Strokes Equation (RANSE) [12, 26]. Potential flow is governed by Laplace equation and discretised using body surface and free surface panels [27]. It is a very useful algorithm, especially in the analysis of free surface flows [24]. RANSE are used to solve viscous fluid flows and able to represent complex free surfaces, which enables it to accurately evaluate total resistance, propulsion, appendages and added resistance. A key advantage of the RANSE methods is its ability to capture global and localised wave patterns as well as effects of viscosity at full scale [28]. Nonetheless, a common criticism of this method is the high computational resources and time needed to perform an evaluation. In addition, the quality of results obtained differs significantly depending on the settings, user's experience and software provider [24].

Despite the array of methods available, they are not widely adopted in the marine and offshore community. Crafting of new hull forms remains largely a tedious trialand-error process where most new designs are created through modifications of existing ones based on the designer's experience. This, we believe, is primarily due to the lack of an integrated framework which is capable of (i) capturing designers' experience and knowledge, (ii) translating them into effective problem representations, (iii) efficiently exploring the design search space for new, innovative and optimal design solutions. Key challenges and bottlenecks in SBD are discussed in detail in the next section.

\section{Key CHALLENGES AND OPPORTUNITIES IN} Simulation-BASED Hull Form DESIGN OPTIMISATION

Despite the benefits that SBD offers in hull form design optimisation, its take-up rate in the marine and offshore vessel design space remains limited. Recent developments in optimisation and performance evaluation techniques, coupled with advancements in high performance computing (HPC) have enabled simulationbased design optimisation to be applied successfully in the design of marine and offshore vessels. The key challenges are discussed with potential gaps for improvements and their possible solutions highlighted as follow:

\section{A. Geometry Modelling and Shape Transformation}

Traditional CAD approach such as splines and NURBS do not work well for complex shapes - difficult to express in admissibility conditions (tangentially for complex shapes). This often results $\mathrm{CAD}$ failures and overestimating of the dimensionality of complex shapes [29]. In some cases, the mathematical definition can be manually manipulated. However, this is an extremely tedious and complex process of trial-and-error where individual vertices must be moved to achieve a smooth surface each time any modification is made [30]. While NURBS are most widely used in geometric modelling of ship hull form, they suffer drawbacks such as large quantity of control points and complications during surface fairing [1]. Furthermore, the resulting geometry is constrained in that designers are not able to adjust control points to achieve the desired shapes [31]. Therefore, there is a need for better geometry definition methods that allows design flexibility and can be representative enough for the selected optimiser and solvers to code and decode easily. One possible approach is by introducing a new spline-based design scheme, such as the partial shape preserving (PSP)-spine basis function [32] which can be used for preserving partial shape when blended with different existing designs.

While most modification methods are fast to execute and effective in achieving a smooth hull form, their capacity to explore the search space for optimum design is often limited. As such, the shape to be transformed is limited and optimal results are not guaranteed. A more robust, shape manipulation method that can link seamlessly to the optimiser would therefore be an ideal solution to alleviate this limitation.

\section{B. Optimisation}

The major challenge for most hull form optimisation is the development of a fully automated optimisation process [33], where key processes such as geometry representation, shape variation and performance evaluation can be integrated in a seamless manner. Numerical solution usually varies from user and can produce errors arising from discretization [33]. A common problem in existing optimisation algorithm applied to hull form design is the lack of a robust and effective way to produce meaningful and feasible solutions [34]. This in part is due to the search mechanisms used. However, a 
larger issue lies in the lack of an effective and accurate mapping between the phenotype and genotype to accurately represent the problem. It is a crucial that a large solution set can be effectively represented.

Whilst it is more accurate, direct solver are impractical and time consuming to be applied in hull form optimisation. Advance solvers such as heuristic solvers in this case can help to improve the efficiency drastically but they cannot guarantee to obtain the optimal solution [11]. Approximation techniques or surrogate model can also be used to improve the overall computational cost without over-compromising accuracy of optimisation solution. In performing hull form optimisation, some commonly used approximation techniques include Kriging method [35], artificial neural networks [36] and polynomial response surface methodology (RSM) [37]. Another promising approximation technique in hull shape optimisation is Karhunen-Lo'eve expansion (KLE), which can be used effectively to reduce design space dimension while allowing high degree of geometry modification [38].

Furthermore, although ship design is a multidisciplinary problem consisting of multiple design specifications, attention has been largely focused on solving it as a single objective problem [39]. Unfortunately, real-world engineering applications encompass multi-parameters and disciplines, where improvement in one specific aspect could potentially worsen another [10]. To advance SBD tools, one needs to also consider multi-disciplinary design specifications such as fluid structure interaction or hydro-elasticity.

\section{Performance Evaluation}

Investigating ship hydrodynamics is practically challenging. This is due to the fact that computing viscous resistance requires very fine grids to be modelled around the ship hull [1]. CFD based RANSE method are effective in accurately predicting the fluid dynamics, however, are computationally expensive when applied in hull form optimisation. Advance HPC and parallel computing can be utilise in this case to reduce the time for performance evaluation using CFD significantly. Some RANSE solvers in this case, allow the computation to start from an arbitrary approximation. It helps to save computation time by leveraging on existing flow field approximation for subsequent computation while initial computation are in progress [28].

Results obtained from potential flow or CFD may not be as accurate as compared to model testing, where unknown phenomenon can arise and missed out in the numeric calculation. However, recent advances in CFD had improved in accuracy tremendously and it definitely can provide some good indication during initial design stage and can be used to narrow down to few 'optimum' design before final model testing.

\section{Other Relevant Factors}

There are common skepticisms within ship design firms and shipyards on the use of optimisation and CFD procedures in the design process. This is partly due to the lack of expert knowledge on the concept behind the methods as well as sufficient training to use the tools. In some cases, this is directly link to the underlying mechanisms of the methods, for example, in 'black-box' or model-free approaches where users have little or no understanding of how results are obtained. Results obtained from hull form design optimisation can also vary widely depending on the designer's experience and the algorithms used. In particular, for new or novel designs, unknown phenomenon may be neglected in the simulation resulting in erroneous designs. A potential solution to reduce the dependency and experience of designer here is to incorporate design knowledge extraction techniques such as data-mining techniques [37] or machine learning. When incorporated into hull form design framework, these techniques can help to acquire useful design knowledge about the hull form.

There are many commercial software that offers good SBD solution but they have usually rigid system configuration that does not allow users to customise to their specific needs. As such, inter-portability of models between different software can be an issue and even if they are compatible, much effort is still needed to clean-up and modify the transferred models. An opportunity here is the development of hull surface design tool that enable data to be exchanged between different systems using commonly supported representation with high degree of accuracy [29].

A SWOT analysis, summarising the aforementioned challenges and opportunities, is presented in Figure 2.

\begin{tabular}{|l|l|}
\hline \multicolumn{1}{|c|}{ (S) Strengths } & \multicolumn{1}{c|}{ (W) Weaknesses } \\
\hline $\begin{array}{l}\text { - Formal hull form optimisation } \\
\text { procedure instead of ad-hoc } \\
\text { design improvement } \\
\text { - More efficient and cost } \\
\text { effective as compared to model } \\
\text { testing }\end{array}$ & $\begin{array}{l}\text { - Development of fully automated } \\
\text { loop still lacking } \\
\text { - Current numerical methods } \\
\text { cannot guarantee optimal solution } \\
\text { - Computational expensive } \\
\text { optimised hull form design } \\
\text { especially for CFD calculations }\end{array}$ \\
\hline $\begin{array}{l}\text { (O) Opportunities } \\
\text { robustness and practicability }\end{array}$ \\
\hline $\begin{array}{l}\text { - Advance computation } \\
\text { methods such as approximation } \\
\text { multi-disciplinary, data-mining, } \\
\text { machine learning } \\
\text { - Development of more } \\
\text { automated and robust } \\
\text { techniques to improve overall } \\
\text { usablity and efficiency of hull } \\
\text { form design optimisation }\end{array}$ & $\begin{array}{l}\text { - Accuracy of result depends } \\
\text { areatly on designer experience } \\
\text { and input } \\
\text { - Unknown phenomenon for } \\
\text { novel hull design } \\
\text { - 'Black-box' function }\end{array}$ \\
\hline
\end{tabular}

Figure 2. SWOT analysis

There is no doubt that both SBD and hull form optimisation has come a long way. Nonetheless, the above challenges would need to be adequately addressed before these techniques can be more widely accepted and implemented in the design of marine and offshore vessels.

\section{Case Study of Simulation-Based Hull Form DESIGN OPTIMISATION}

To illustrate the application of SBD to hull form design optimisation, a case study of a 180 meters passenger ferry vessel was carried out using NAPA design solutions. The objective is to modify the shape of an existing bulbous bow design in order to improve the wave making resistance. Unlike conventional manual SBD 
method where hull form optimisation are carried out individually and manually, purpose of this study is to demonstrate the practicability and efficiency of a fully automated hull form optimisation solution by integrating the different processes into one common environment. As such, this procedure was carried out using manager function of NAPA and the steps are given as follows:

1) The vessel was first modelled in NAPA hull modelling tool. For shape transformation, free-form deformation (FFD) technique was selected. The bulbous bow shape was modified simply by creating boundary box and extending the length by 3 metres toward forward direction (fig 3).

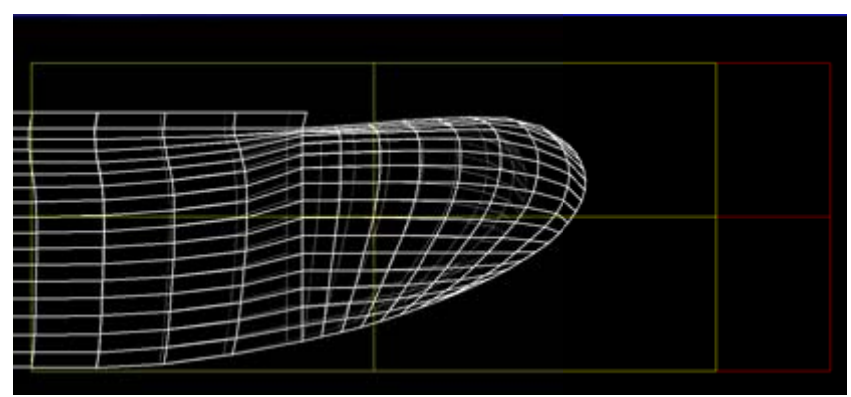

Figure 3. Free Form Deformation (FFD) of bulbous bow area

2) The hull surface mesh was created under NAPA panalisation manager, followed by evaluation of hull panelised model using potential flow method.

3) Selected MOGA for optimisation with setting of 10 population, 20 generations and encoding scheme linked to FFD. Total time taken for calculation was 30 minutes using standard intel Core i7-4702 CPU workstation. Optimum design was subsequently obtained demostrating a slight reduction of $3 \%$ to forward end resistance coefficient.

4) The wave and pressure profile of hull model with modified bulbous bow are then exported to postproccessor ParaView as shown in figure 4.

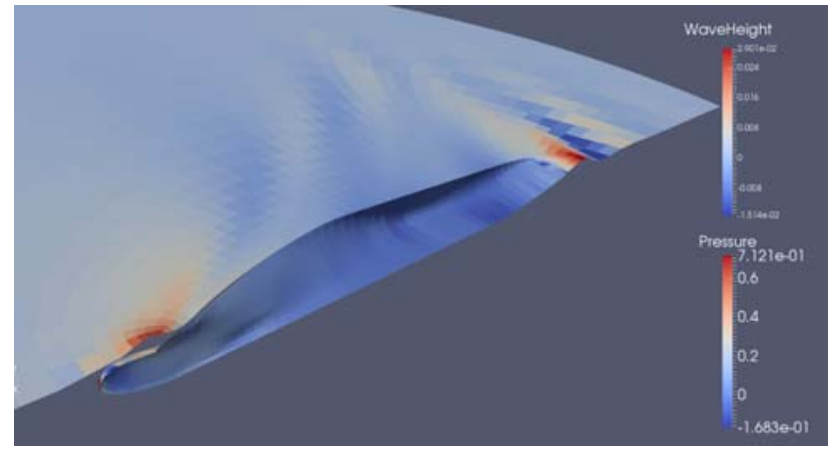

Figure 4. Wave profile of hull model with modified bow

The following findings have been observed from the above case study:

- This study has demonstrated the efficiency and effectiveness of an automated hull form optimisation process using FFD, MOGA and CFD techniques. Although the improvement is marginal, it provides a good indication of how the hull should be modified to further improve the overall performance. This is useful to the designer and can be used as a starting point to further investigations using more detailed procedures such as RANSE.

- Hull geometry needs to be properly modelled and adequately smooth. Failing to do so will result in panelisation error as well as evaluation results. It was observed geometry modelling of ship hull was one of the most time consuming task.

- $\quad$ Free-from deformation was applied easily and it is capable of producing rather smooth curve without discontinuity in the geometry. However, it was noted the coordinate to move greatly depends on the experience of designer, which makes it difficult to locate the Pareto optimal.

- In order to increase the chance of obtaining the Pareto optimum set, more shape variations should be tested and used as inputs for the optimisation. However, this tends to be a 'trial and error' process and again depends greatly on the designer's experience for input. This thereby presents another area for improvement.

\section{CONCLUSION}

Due to stringent environmental regulations and volatile fuel prices, marine and offshore vessels are now expected to be more eco-friendly and fuel efficient. In this context, simulation-based hull form design optimisation is gaining increasing attention and importance. It has been demonstrated to be a very efficient and cost-effective tool as compared to ad-hoc manual design improvements and model testing. Key processes in simulation-based hull form design consist of geometry modelling and shape transformation, optimisation and performance evaluation. Main challenges in performing hull form optimisation include scepticism due to 'black-box' functions, high computational costs, automated optimisation loop, for example. Recent developments in advance computation techniques and more powerful computers have presented multiple opportunities such as multi-disciplinary optimisation, machine learning, approximation methods, and optimised hull form design based on variable speed or multi-draft. It has been demonstrated in the case study of bulbous bow optimisation that formal hull form optimisation can be applied successfully using combination of potential flow code method, Free-Form Deformation and MOGA techniques, to improve forward end resistance of ferry vessel. By addressing the challenges and further developing on the opportunities presented, it is envisaged that simulation-based hull form optimisation can become more accepted and widely applied in marine and offshore vessel design.

\section{ACKNOWLEDGMENT}

The authors would like to thank NAPA for granting the usage of their 'eco-design' packages and support provided for the case study applied in this paper.

\section{REFERENCES}


[1] R. Sharma, Tae-wan Kim, Richard Lee Storch, Hans (J.J.) Hopman, Stein Ove Erikstad, "Challenges in computer applications for ship and floating structure design and analysis," Comput. Aided Design, vol. 44, 2012, pp. 166185.

[2] Emilio F. Campana, Daniele Peri, Yusuke Tahara, Frederick Stern, "Shape optimization in ship hydrodynamics using computational fluid dynamics," Comput. Methods Appl. Mech. Engrg. 196, 2006, pp. 634651.

[3] Shengzhong Li and Feng Zhao, "An innovative hull form design technique for low carbon shipping," J. of Shipping and Ocean Eng., 2012.

[4] Soonhung Han, Yeon-Seung Lee, Young Bok Choi, "Hydrodynamic hull form optimization using parametric models," J. Mar. Sci. Technol. ,2012.

[5] H. Lackenby, "On the systematic geometrical variation of ship forms," Trans. of the royal inst. of naval architects, 1950.

[6] Marcus Bole, "Interactive hull form transformations using curve network deformation," Conf. Comput. and IT applicat. in Maritime Ind. (COMPIT), 2010.

[7] Claus Abt, Stefan Harries, Justus Heimann, Henning Winter, "From redesign to optimal hull lines by means of parametric modeling," Conf. Comput. and IT applicat. in Maritime Ind. (COMPIT), 2003.

[8] Francisco Pérez, José A. Suárez, Juan A. Clemente, Antonio Souto, "Geometric modelling of bulbous bows with use of non-uniform rational B-spline surfaces," J. Mar. Sci. Technol., 2007, pp. 83-94.

[9] Daniele Peri, Emilio F. Campana, Manivannan Kandasamy, Seng Keat Ooi, Pablo Carrica, Frederick Stern, Phil Osborne, Neil Macdonald, Nic de Waal, "Potential flow based optimization of a high speed, foilassisted, semi-planning catamaran for low wake," in $10^{\text {th }}$ Int. Conf. on Fast Sea Transportation, Athens, Greece, 2009.

[10] Yusuke Tahara, Daniele Peri, Emilio Fortunato Campana, Frederick Stern, "Computational fluid dynamics-based multiobjective optimization of a surface combatant using a global optimization method," J. Mar. Sci. Technol., 2008, pp. 95-116.

[11] Slawomir Koziel and Xin-She Yang, "Computational Optimization, Methods and Algorithms," Springer-Verlag Berlin Heidelberg, 2011.

[12] Scott Perival, Dane Hendrix, Francis Noblesse, "Hydrodynamic optimization of ship hull forms," Applied Ocean Research 23, 2001, pp. 337-355.

[13] Kazuo Suzuki, Hisashi Kai, Shigetoshi Kashiwabara,"Studies on the optimization of stern hull form based on a potential flow solver," J. Mar. Sci. Technol., 2005, pp. 61-69.

[14] V. Anantha Subramanian, G. Dhinesh, J.M. Deepti, "Resistance optimisation of high speed catamarans," Canada's Arctic, Vol. 1, No. 1, 2006, pp. 69-82.

[15] D. Peri, M. Rossetti, E.F. Campana, "Design optimization of ship hulls via CFD techniques," J. of Ship Research, Vol. 45, 2001, pp. 140-149.

[16] C. Cinquini, P. Venini, R. Nascimbene and A. Tiano, "Design of river sea ship by optimization," Struct. Multidisc. Optim. 22, 2001, pp. 240-247.

[17] Ho-Hwan Chun, "Hull form parameterization technique with local and global optimization algorithm," Proc. of The Int. Conf. on Marine Technol., Dhaka, Bangladesh, 2010.

[18] Matteo Diez, Andrea Serani, Umberto Iemma, Emilio F. Campana, "A fish shoal algorithm for global derivativefree simulation-based ship design optimization," 17th Numercial Towing Tank Symp., 2014.

[19] T. Ray, R.P. Gokarn, O.P. Sha, “A global optimization model for ship design," Comput. in Ind. 26, 1995, pp. 175192.
[20] Dunja Matulja, Roko Dejhalla, "Hydrodynamic optimisation of forepart of ship," Proc. of XX Symp., Zagreb, Croatia, 2012.

[21] Grzegorz Mazerski, "Optimisation of FPSO's main dimension using Genetic Algorithm," Proc. of the ASME 31 st Int. Conf. on Ocean, Offshore and Arctic Eng., Rio de Janeiro, Brazil, 2012.

[22] Sheng Huang, Wanlong Ren, Chao Wang, and Chunyu Guo, "Application of an improved particle swarm optimization algorithm in hydrodynamic design," ICSI 2013, Part I, LNCS 7928, 2013, pp. 225-231.

[23] Emilio Fortunato Campana, Giampaolo Liuzzi, Stefano Lucidi, Daniele Peri, Veronica Piccialli, Antonio Pinto, "New global optimization methods for ship design problems," Optim, Eng., Vol.10, 2009, pp. 533-555.

[24] Volker Betram, "Appropiate tools for flow analyses for fast ships," 4th Int. Conf. High-Performance Marine Vehicles (HIPER), Naples, 2008, pp. 1-9.

[25] Frederick Stern, Jianming Yang, Zhaoyuan Wang, Hamid Sadat-Hosseini, Maysam Mousaviraad, Shanti Bhushan, Tao Xing, "Computational ship hydrodynamic: Nowadays and way forward," $29^{\text {th }}$ Symp. on Naval Hydrodynamics, Gothenburg, Sweden, 2012.

[26] Ruosi Zha, Haixuan Ye, Zhirong Shen, Decheng Wan, "Numerical study of viscoius wave making resistance of ship navigation in still water," J. Marine Sci. Appl., vol. 13, 2014, pp. 158-166.

[27] Horst Nowacki, "Hydrodynamic design of ship hull shapes by methods of computational fluid dynamics," Progress in Ind. Math. at ECMI , 1996, pp. 232-251

[28] Karsten Hochkirch, Benoit Mallol, "On the importance of full-scale CFD simulations for ships," Conf. Comput. and IT applicat. in Maritime Ind. (COMPIT), 2013.

[29] D. Bülent Danışman, Ömer Gören, Mustafa Insel, Mehmet Atlar, "An optimisation study for bow form of high speed catermarans," Marine Technol., Vol. 38, No. 2, 2001.

[30] Marcus Bole, Byung-Suk Lee, "Integrating parametric hull generation into early stage design," Ship Technol. Research, Vol 53, 2006, pp. 115-137.

[31] Herbert J. Koelman, Bastiaan N. Veelo, "A technical note on the geometric representation of a ship hull form," Comput. Aided Design, vol. 45, 2013, pp. 1378-1381.

[32] Qingde Li, Jie Tian, "Partial shape-preserving splines," Comput. Aided Design, vol. 43, 2011, pp. 394-409.

[33] K.V. Kostas, , A.I. Ginnis, C.G. Politis, P.D. Kaklis, "Ship-hull shape optimization with a T-spline based BEMIsogeometric solver," Comput. Methods Appl. Mech. Engrg., 2014.

[34] Yusuke Tahara, Daniele Peri, Emilo Fortunato Campana, Frederick Stern, "Single and multiobjective design optimization of a fast multihull ship: numerical and experimental results," J. Mar. Sci. Technol., vol. 16, 2011, pp. 412-433.

[35] Daniele Peri and Federica Tinti, "A multistart gradientbased algorithm with surrogate model for global optimization" Commun. in Appl. and Ind. Math., vol 3, no $1,2012$.

[36] Kourosh Koushan, "Automatic hull form optimisation towards lower resistance and wash using artificial intelligence," Int. Conf. on Fast Sea Transportation, 2003.

[37] Shinkyu Jeong and Hyunyul Kim, "Development of an efficient hull form design exploration framework," Math. Problems in Eng., vol. 2013.

[38] Matteo Dieza, Emilio F. Campana, Frederick Stern, "Design-space dimensionality reduction in shape optimization by Karhunen-Lo'eve expansion," Comput. Methods Appl. Mech. Engrg., 2014.

[39] Daniele Peri, Antonio Pinto, Emilio F. Campana, "Multiobjective optimisation of expensive objective functions with variable fidelity models," Large-Scale Nonlinear Optim, vol. 83, 2006. 
